\title{
An Estimate of the Live Time of Optical Measurements of Air Showers at the South Pole
}

\author{
Segev BenZvi ${ }^{* a}$ and Stephen Drury ${ }^{a}$ \\ ${ }^{a}$ Department of Physics and Astronomy, University of Rochester, Rochester, NY, USA \\ Email: sybenzvi@pas.rochester.edu
}

\begin{abstract}
The recent observation of $\mathrm{PeV}$ neutrinos at the IceCube Neutrino Observatory has revived interest in deploying large-exposure optical air shower detectors at the South Pole. The main challenge for such detectors, which were last deployed at the Pole in the 1990s, is the poor atmospheric conditions prevalent during austral winter. To investigate the clarity of the atmosphere we have studied archival weather data from the Atmospheric Research Observatory at the South Pole. Using several years of winter measurements with radiosondes and the MPLNET $532 \mathrm{~nm}$ lidar station, we obtained estimates of the cloud coverage and optical clarity of the site. Based on past data, it is likely that an optical detector will achieve $<45 \%$ uptime during the dark period at the Pole, making the total annual live time comparable to the live time of optical air shower detectors in the middle latitudes.
\end{abstract}

The 34th International Cosmic Ray Conference,

30 July- 6 August, 2015

The Hague, The Netherlands

* Speaker. 


\section{Introduction}

The discovery of astrophysical neutrinos by the IceCube collaboration [1, 2, 3] has created interest in deploying large-scale air shower detectors at the South Pole. The detection of air shower particles in time coincidence with a neutrino observation inside the IceCube detector can provide an excellent veto of the downgoing atmospheric neutrinos produced by cosmic rays. Although the IceCube Neutrino Observatory currently has a "veto layer" in the form of the $1 \mathrm{~km}^{2}$ IceTop air shower array, the array can only be used to veto atmospheric neutrinos from a limited set of near-vertical shower geometries. Therefore, the extension of the surface array to allow coverage of a large fraction of the southern sky is well motivated [4].

While an extension of IceTop is the most likely path to a very large-scale air shower detector at the Pole, other technologies such as air Cherenkov telescopes are under consideration [5]. Air Cherenkov detectors were previously proposed [6] and operated [7] at the Pole in the context of gamma-ray astronomy. These telescopes can only operate during dark periods (about $40 \%$ of the year at the South Pole) but could perform a dual role as an air shower veto and as a gamma-ray detector.

Unfortunately the livetime of air Cherenkov telescopes is also limited by weather conditions such as clouds, precipitation, and high winds. Even within Antarctica, the South Pole is a particularly harsh environment for astronomical observations, suffering from extreme cold, strong wind, and being located several hundred meters inside the particle boundary layer [8].

To evaluate the feasibility of deploying and operating air Cherenkov detectors at the South Pole, we have studied archival weather data from Antarctica, focusing specifically on atmospheric clarity (clouds and aerosols). In the next section we describe the data used to estimate cloud coverage at the South Pole during the dark period of austral winter, followed by a discussion of our results and a comparison to previous observations.

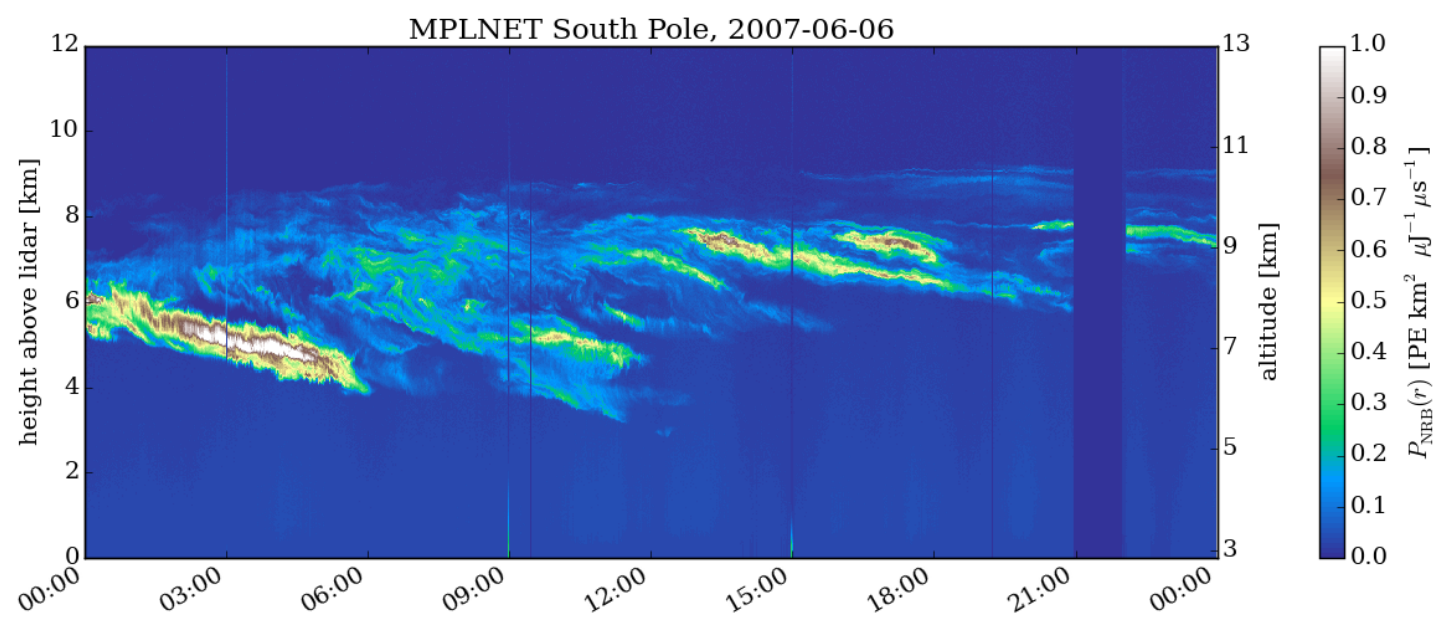

Figure 1: Time series of 1-minute vertical atmospheric profile measurements recorded by the MPLNET lidar at the Amundsen-Scott Station, 6 June 2007. The data are expressed in terms of the normalized relative backscatter $P_{\mathrm{NRB}}$. Large values of $P_{\mathrm{NRB}}$ indicate the presence of clouds and aerosols. 


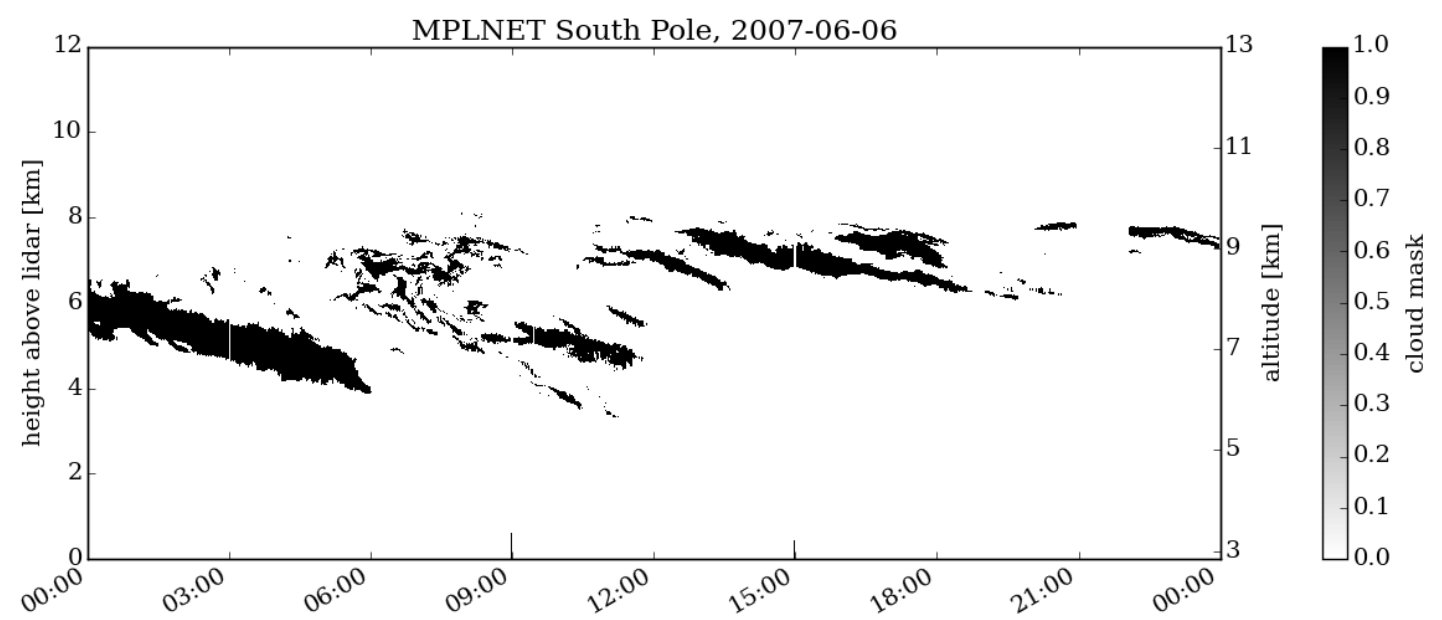

Figure 2: Cloud mask produced by thresholding the lidar returns in Fig. 1.

\section{Lidar Data at the South Pole}

Although the South Pole is one of the most remote sites in the world, there exist decades of continous observations of weather conditions from this location. During the past 10 years, meteorological observations at the Pole have been supplemented with data from the Micropulse Lidar Network, or MPLNET [9]. MPLNET is a network of autonomous and semi-autonomous lidar stations located around the world. The lidar at the Pole is deployed in the Atmospheric Research Observatory (ARO) at the Amundsen-Scott Station.

The MPLNET lidar at ARO is well-suited for observations of clouds and aerosols. It operates by pulsing a vertically-oriented $532 \mathrm{~nm}$ laser beam into the atmosphere and recording the backscattered laser light using a mirror and photosensor next to the laser [10]. The laser power $P$ observed from a scattering site at height $r$ above the lidar station is given by

$$
P(r)=\frac{C \cdot E \cdot O(r) \cdot \beta(r) \cdot T^{2}(r)}{r^{2} D(P)}+\frac{a(r, E)}{D(P)}+\frac{b}{D(P)},
$$

where $C$ is a system constant, $E$ is the laser energy per pulse, $O(r)$ is a geometry factor describing the overlap between the laser beam and mirror field of view, $\beta$ is the backscatter coefficient of the air at distance $r, D(P)$ is the deadtime of the photosensor, and $T^{2}(r)$ is the two-way transmission of the beam from the laser to the scattering point $r$ and back to the receiver:

$$
T^{2}(r)=\exp \left[-2 \int_{0}^{r} \sigma\left(r^{\prime}\right) d r^{\prime}\right]
$$

The quantity $\sigma$ is the attenuation coefficient of air, which (neglecting absorption) is just the volume scattering coefficient. The additional terms $a(r, E)$ and $b$ in eq. (2.1) are noise due to PMT afterpulsing and sky background, respectively.

The presence of clouds and aerosols increases the scattering coefficients $\beta$ and $\sigma$ of the air, and so clouds appear as strong reflections and echoes in the observed laser power (see Fig. 1). The MPLNET project provides a noise-subtracted, range-corrected version of $P(r)$ for public download. 
This quantity, called the normalized relative backscatter

$$
P_{\mathrm{NRB}}(r)=C \cdot \beta(r) \cdot T^{2}(r),
$$

is also corrected for drifts in the energy of the laser and the geometric overlap between the mirror and the laser beam. $P_{\mathrm{NRB}}$ depends only on the backscatter coefficient $\beta$, the volume scattering coefficient $\sigma$, and the undetermined system response constant $C$.

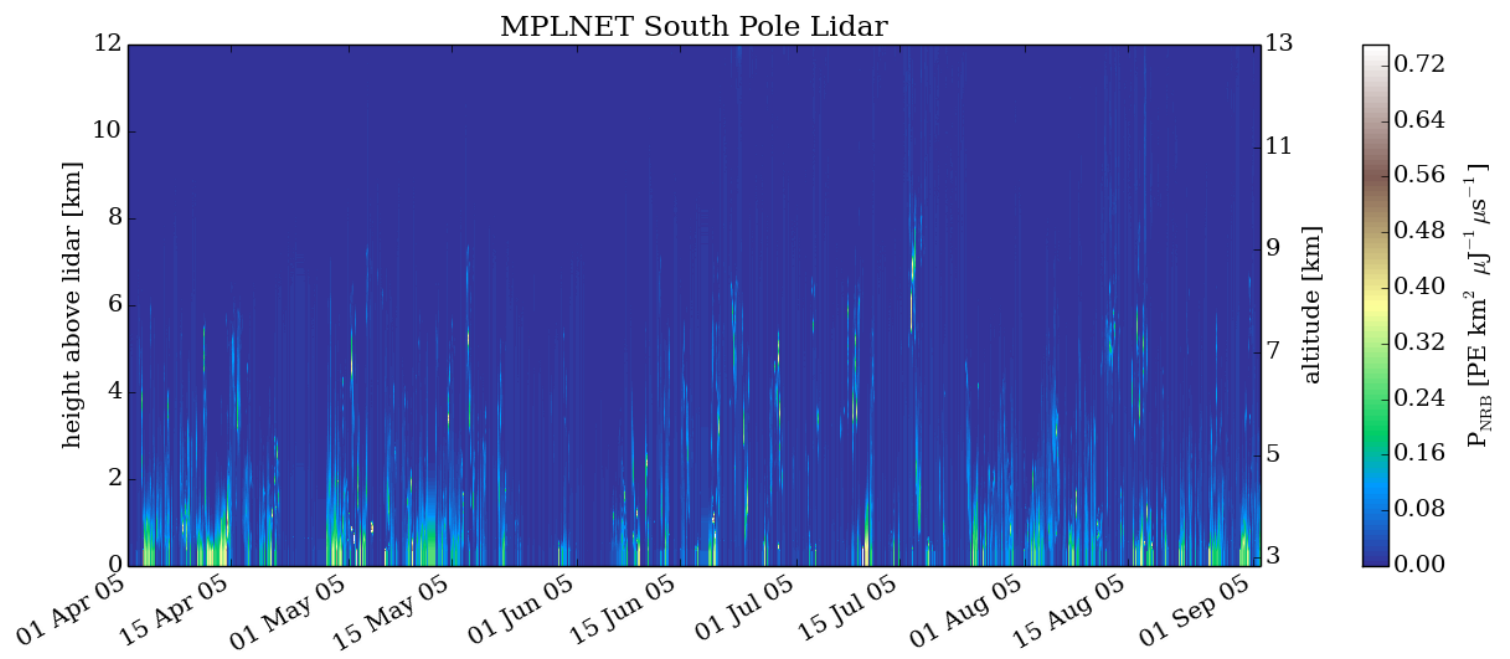

Figure 3: Daily vertical atmospheric profiles from the MPLNET lidar covering the austral winter of 2005.

\section{Cloud Detection Analysis}

In the presence of a weak return lidar power with a poor signal to noise ratio, there exist several algorithms for the automatic detection of cloud boundaries [11]. However, our interest is mainly in the detection of clouds in the troposphere, a region of high signal to noise where most of the Cherenkov light in an air shower is produced. Clouds in the lower troposphere are of particular interest because they can attenuate light between air showers and the detector. We found that a simple threshold on $P_{\mathrm{NRB}}$ is sufficient to detect the optically thick clouds in the troposphere that can affect observations with air Cherenkov telescopes.

The detection algorithm works as follows. First, a cloud mask is produced by applying a threshold to $P_{\mathrm{NRB}}$. For example, Fig. 2 shows the clouds identified by setting a threshold of $0.2 \mathrm{PE} \mathrm{km}{ }^{2} \mu \mathrm{J}^{-1} \mu \mathrm{s}^{-1}$ on the data in Fig. 1. The threshold was optimized by looking at several months of data from 2004, comparing cloudy days to clear days with and without subtraction of the molecular scattering component of the atmosphere.

Once a cloud mask was produced, we looked for spatially and temporally contiguous regions of cloud, e.g., removing any region that is cloudy for $<15$ minutes. All remaining regions were classified as clouds. To produce a conservative estimate of the base height for each cloud (i.e., the altitude of the lower edge of the cloud), we drew a bounding box around each identified cloud and reported the lower horizontal edge of the box as the altitude of the cloud base. 


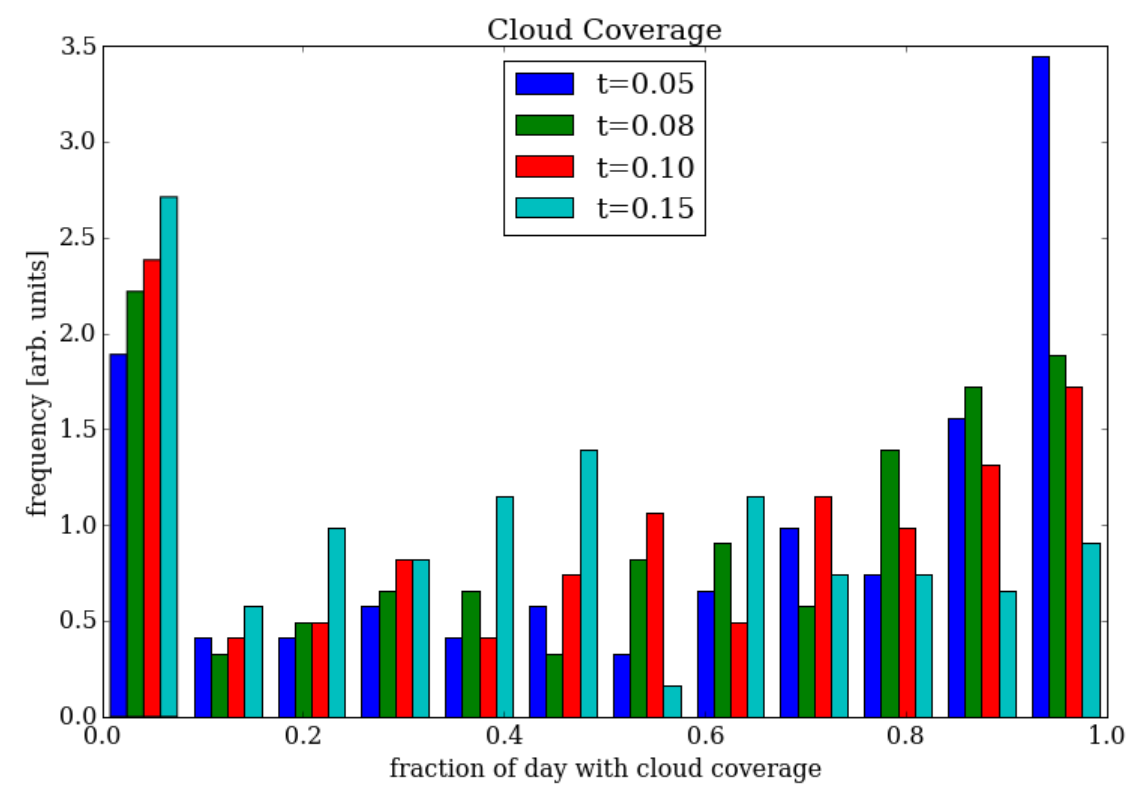

Figure 4: Cloud coverage fraction at the South Pole, obtained with four different thresholds on $P_{\mathrm{NRB}}$. Small clouds which cover $<15$ minutes in the lidar data were removed from the sample before producing the coverage histogram.

\section{Cloud Coverage and Base Height Distribution}

Figure 3 shows the atmospheric profiles from the MPLNET station at ARO from April to September 2005, which roughly covers the dark period of austral winter. Visual inspection of the figure shows alternating periods of clear and cloudy conditions, with the bulk of the clouds and heavy aerosols appearing near ground level.

To automatically identify the cloud coverage and base height distribution during this period, we applied several thresholds on $P_{\mathrm{NRB}}$. The thresholds are too high to catch optically thin aerosols, such as the "diamond dust" which causes the areola of scattered light around bright light sources at the Pole, but they are sufficient to select regions with optically thick clouds.

A histogram of cloud coverage from the 2005 and 2006 austral winters is shown in Fig. 4. Four separate thresholds on $P_{\mathrm{NRB}}$ were chosen (ranging by a factor of 3 ) to estimate systematic uncertainties in the choice of threshold. The data are expressed in terms of the fraction of each day with clouds, with any clouds covering $<15$ minutes removed from the sample. While the results vary depending on the choice of threshold, in all cases we observe a fairly bimodal distribution, with the most common conditions being completely overcast or completely clear in each 24-hour period.

Figure 5 shows the distribution of the base heights of clouds observed with two thresholds on $P_{\mathrm{NRB}}$ and two minimum duration lengths for selecting clouds (15 minutes and 20 minutes). Most of the optically thick clouds and aerosols observed at the Pole occur within $1 \mathrm{~km}$ of the surface of the ice sheet; these clouds would produce significant down time for an optical detector on the surface. The clouds between 1-4 km would not necessarily preclude telescope operations, but would constrain measurements of air showers if observations of shower maximum are required. Clouds 


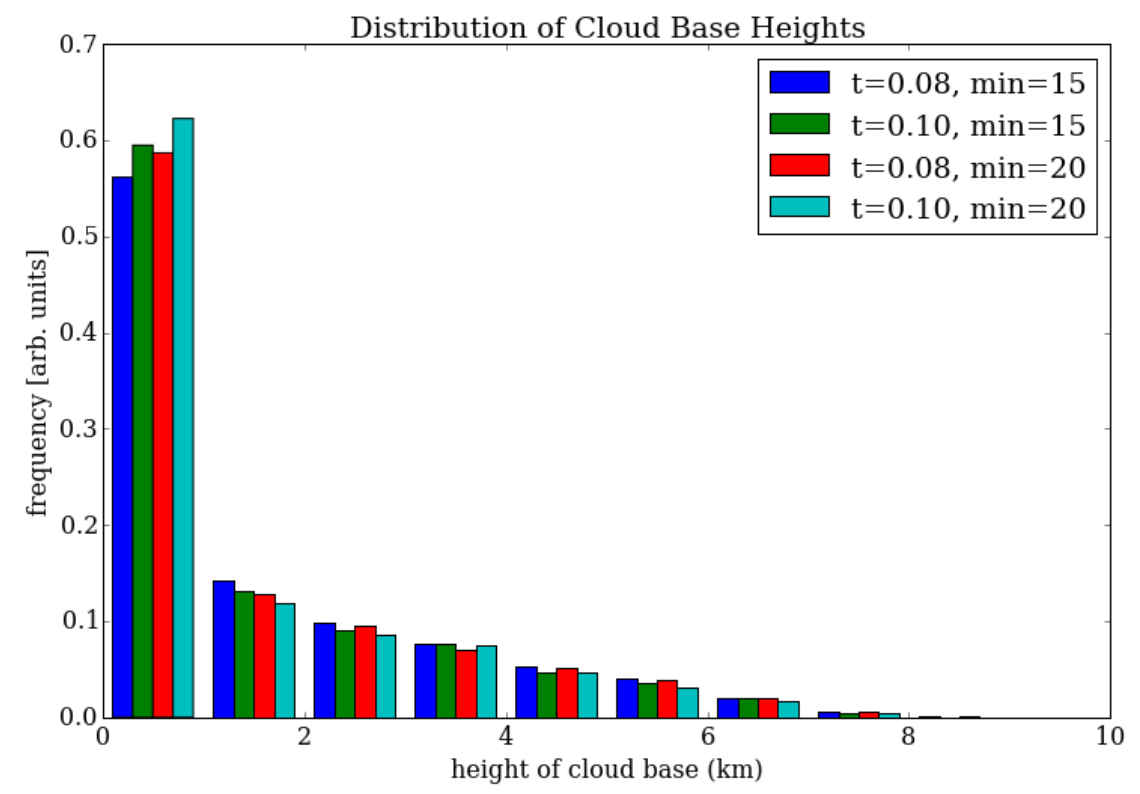

Figure 5: Distribution of the heights of cloud bases at the South Pole, expressed in kilometers above the surface of the polar ice sheet.

above $4 \mathrm{~km}$ could block observations of shower maximum for some geometries, but otherwise should be considered good operating conditions.

The fraction of days without significant cloud coverage (defined as $<30 \%$ coverage per day) or with high-altitude clouds is $<45 \%$. This estimate is in agreement with previous IR radiometry measurements performed at the Pole [12].

\section{Conclusions}

If we account for the number of days with relatively little cloud coverage at the Pole, defined as $<30 \%$ coverage per day, combined with all periods that have clouds $>4 \mathrm{~km}$ above the surface, we find that the total period suitable for observations of air showers (with shower maximum) ranges between $35 \%$ and $55 \%$. The uncertainty depends on the choice of threshold for $P_{\mathrm{NRB}}$. Since the dark period at the South Pole lasts about $40 \%$ of the year, we expect that the total livetime of an air Cherenkov telescope at the pole will be $<20 \%$, similar to the livetime of telescopes in the middle latitudes.

If we relax the demand that shower maximum is observed - for example, observation of the shower maximum is not needed for the telescope to act as a cosmic-ray air shower veto - then the telescope livetime will increase because data-taking can occur on during periods with more cloud coverage. The maximum expected livetime during the dark period would be $\sim 60 \%$ in this case.

\section{Acknowledgments}

This work was supported in part by a grant from the REACH program at the University of Rochester. The NASA Micro-Pulse Lidar Network is funded by the NASA Earth Observing System and Radiation Sciences Program. We thank 
the MPLNET PI E. J. Welton (NASA GSFC) and MPLNET staff T. Berkoff, J. Campbell, and J. Spinhirne (NASA GSFC) for their efforts in establishing and maintaining the South Pole site.

\section{References}

[1] IceCube Collaboration, M. Aartsen et al., First observation of PeV-energy neutrinos with IceCube, Phys.Rev.Lett. 111 (2013) 021103, [arXiv:1304.5356].

[2] IceCube Collaboration, M. Aartsen et al., Evidence for High-Energy Extraterrestrial Neutrinos at the IceCube Detector, Science 342 (2013) 1242856, [arXiv : 1311 . 5238].

[3] IceCube Collaboration, M. Aartsen et al., Observation of High-Energy Astrophysical Neutrinos in Three Years of IceCube Data, Phys.Rev.Lett. 113 (2014) 101101, [arXiv: 1405.5303 ].

[4] IceCube Collaboration, J. Auffenberg, Motivations and Techniques of a Surface Detector to Veto Air Showers for Neutrino Astronomy with IceCube at the Southern Sky, in Proc. 34th ICRC, (The Hague, The Netherlands), August, 2015.

[5] IceCube Collaboration, J. Schumacher and J. Auffenberg, Design Study of an Air Cherenkov Telescope for Efficient Air-Shower Detection at $100 \mathrm{TeV}$ at the South Pole, in Proc. 34th ICRC, (The Hague, The Netherlands), August, 2015.

[6] R. Morse, A South Pole facility to observe very high-energy gamma-ray sources, Nucl. Phys. Proc. Suppl. 14B (1990) 61-67.

[7] J. E. Dickinson et al., A new air-Cherenkov array at the South Pole, Nucl. Instrum. Meth. A440 (2000) 114-123.

[8] M. G. Burton, Astronomy in Antarctica, Astron.Astrophys.Rev. 18 (2010) 417-469, [arXiv:1007.2225].

[9] “The Micropulse Lidar Network (MPLNET).” http://mplnet.gsfc.nasa.gov, 2015. Accessed: 2015-07-10.

[10] J. Campbell, D. Hlavka, E. Welton, C. Flynn, D. Turner, J. Spinhirne, V. Scott, and I. Hwang, Full-Time, Eye-Safe Cloud and Aerosol Lidar Observation at Atmospheric Radiation Measurement Program Sites: Instruments and Data Processing, J. Atmos. Oceanic Technol. 19 (2002) 431-442.

[11] J. Campbell, K. Sassen, and E. Welton, Elevated Cloud and Aerosol Layer Retrievals from Micropulse Lidar Signal Profiles, J. Atmos. Oceanic Technol. 25 (2008) 685-700.

[12] A. Mahesh, V. P. Walden, and S. G. Warren, Ground-Based Infrared Remote Sensing of Cloud Properties over the Antarctic Plateau. Part I: Cloud-Base Heights, J. Appl. Meteor. 40 (2001) 1265-1278. 